\title{
Evaluación del estado de conservación de la vegetación de los bosques de una cuenca heterogénea del Valle de México
}

\author{
Assessing the conservation status of the vegetation in forests \\ of a highly heterogeneous watershed of Mexico Valley
}

\author{
Gabriela Santibañez-Andrade ${ }^{\text {a }}$, Silvia Castillo-Argüero ${ }^{\text {a*, }}$ Yuriana Martínez-Orea ${ }^{\text {a }}$ \\ *Autor de correspondencia: a Universidad Nacional Autónoma de México, Facultad de Ciencias, \\ Departamento de Ecología y Recursos Naturales, Laboratorio de Dinámica de Comunidades, 04510, D.F., México, \\ tel.: 5255 56228222, silcas@ciencias.unam.mx
}

\author{
SUMAMRY
}

Studies that evaluate conservation status of ecosystems in Mexico often lack the integration of some theoretical bases as well as some methodological tools that landscape ecology offers. This situation imposes limitations for the implementation of more adequate management, restoration and conservation strategies. In this study we propose a four stage method in order to characterize the biophysical environment and to estimate the vegetation conservation status in a highly environmentally heterogeneous basin, under strong anthropogenic pressure. The stages were: 1) delimitation and characterization of environmental units based on biophysical conditions, 2) quantification of vegetation structure and composition, 3) classification of the species according to their functional responses, and 4) evaluation of the conservation status based on the community structure and composition. As a result, biophysical units showed differences in richness, diversity, and in the importance index values of native, weed and introduced species. Plant functional groups were identified; they comprised different environmental responses in a conservation gradient in the biophysical units. Through the canonical correspondence analysis, it was demonstrated that composition is mainly related to the light regime, temperature, humidity and some soil conditions. Significant differences in the conservation status of the biophysical units were found. Therefore vegetation composition is an important indicator of the conservation status; its definition helps to generate punctual strategies for conservation planning.

Key words: plant functional groups; non-native species, weeds, temperate forests, Magdalena river watershed.

\section{RESUMEN}

Los estudios que evalúan el estado de conservación de los ecosistemas en México a menudo no integran bases teóricas o herramientas metodológicas que ofrece la ecología del paisaje. Esta situación limita la posibilidad de construir estrategias de manejo más adecuadas, así como acciones de restauración y conservación. En este estudio se propuso un método de cuatro etapas para caracterizar el ambiente biofísico y estimar el estado de conservación de la vegetación en una cuenca con alta heterogeneidad ambiental y fuerte presión antropogénica. Las etapas fueron: 1) delimitación y caracterización de unidades de ambientales en base a las condiciones biofísicas, 2) cuantificación de la estructura y composición de la vegetación, 3) clasificación de las especies de acuerdo a sus respuestas funcionales, y 4) evaluación del estado de conservación basado en la estructura y la composición. Como resultado, las unidades biofísicas tuvieron diferencias en su riqueza, diversidad, valor de importancia de las especies nativas, malezas e introducidas. Se identificaron grupos funcionales de plantas con diferentes respuestas ambientales y que reflejan un gradiente de conservación de las unidades biofísicas. A través de análisis de correspondencia canónica se demostró que esta composición estuvo principalmente relacionada con la luz, temperatura, humedad y algunas condiciones del suelo. Se encontraron diferencias significativas del grado de conservación entre las unidades biofísicas. La composición de la vegetación fue un indicador importante del estado de conservación, lo cual ayudará a generar estrategias de gestión puntuales en la planificación o proceso de conservación.

Palabras clave: grupos funcionales de plantas, especies introducidas, malezas, bosques templados, cuenca del río Magdalena.

\section{INTRODUCCIÓN}

La planeación de la conservación de las zonas forestales debe ser un proceso estructurado con el objetivo de identificar las prioridades y decidir dónde, cuándo y cómo preservar los componentes principales de la biodiversidad. El pro- ceso completo requiere de estudios con un enfoque integral con el fin de informar, analizar y monitorear el estado de conservación de la cubierta vegetal (Thackway et al. 2007).

Para efectos de la planificación, es necesario explorar cómo los tipos o elementos del paisaje influyen sobre la diversidad al generar ambientes que pueden ser aprovecha- 
dos de manera diferencial por las especies (Dummel y Pinazo 2013). Por lo tanto, un estudio diagnóstico debe tener en cuenta dos aspectos importantes; a) la determinación y la caracterización del mosaico ambiental y b) la recopilación de información precisa que sirva como un indicador de la estructura y funcionamiento del ecosistema (Fairbanks y Benn 2000).

La clasificación del paisaje en unidades es una herramienta importante de la biología de la conservación, este proceso debe realizarse con base en sus propiedades ecológicas y físicas (Eswaran et al. 2000). A esta escala general es indispensable describir algunas características generales como: el tipo de suelo, extensión, orientación, pendiente, cobertura vegetal, fisionomía y apertura de dosel, las cuales son útiles para asignar un nivel de conservación. Sin embargo, estas variables generales a menudo no proveen la información específica requerida para la implementación de acciones dirigidas a la preservación de la dinámica del ecosistema (Noss 1990). La información a escala más fina puede complementar esta metodología con la cual se puede analizar si el ensamblaje de las especies locales está influenciado por la calidad del hábitat (Allan 2004). Esta escala permite determinar la biodiversidad local a través de algunos atributos como la riqueza, la diversidad, el valor de importancia, la proporción de especies características, malezas y especies introducidas, así como la presencia de grupos funcionales (Noss 1990).

Una caracterización de los aspectos de la biodiversidad por sí sola no nos indica el estado de conservación. Por lo tanto, en necesario contar con algunos indicadores basados en la estructura y composición para diferenciar un gradiente de conservación (Lindemayer et al. 2000). A nivel de paisaje es importante conocer como las especies difieren en sus respuestas a los cambios ambientales o a agentes de disturbios. Un grupo funcional es un conjunto de especies que exploran la misma clase de recursos ambientales de manera similar, es decir, que sobrepone su nicho ecológico (Westoby y Leishman 1997). En la aproximación de Woodward (1993), la definición de grupos funcionales de acuerdo con un grupo de variables de clima se llaman grupos de respuesta climática (Gomez-Mendoza et al. 2008). El régimen de perturbación altera las condiciones climáticas, por lo tanto, existen algunos grupos de respuesta al régimen de disturbio que se ve reflejado en la composición de especies características, malezas nativas y especies introducidas (Hooper et al. 2002). Estas propiedades son importantes para establecer los tratamientos y las prioridades a nivel de unidades de paisaje (Whilliams et al. 2002).

El establecimiento de malezas nativas y especies introducidas puede ser interpretado como un indicador de la modificación del hábitat (Stenhouse 2004). Las malezas se caracterizan por un crecimiento acelerado, un síndrome de dispersión efectivo y, por lo tanto, un alto índice de abundancia. Estas especies pueden causar efectos indeseables en el sistema como la disminución de la riqueza de especies características del sitio, agotamiento de nutrientes, alelopatía, inclusión de enfermedades y mayor competencia con las especies circundantes (Pysek et al. 2004). Las especies introducidas, se definen como aquellas especies que han sobrepasado sus límites de distribución geográfica con una dispersión muy eficaz o dispersión antropogénica, alterando la estructura y funcionalidad de los ecosistemas receptores (Mooney y Hobbs 2000). Varios estudios discuten la relevancia de resaltar la presencia de especies introducidas y malezas en estudios de comunidades, ya que esto conduce a robustecer significativamente los valores de diversidad nativa (Parkes et al. 2003, Pysek et al. 2004).

En este estudio fue seleccionada la cuenca del río Magdalena ubicada al sur de la Ciudad de México para realizar un diagnóstico del estado de la vegetación, porque: 1) la cuenca es una zona muy heterogénea de 3.100 ha; 2) existe una base de datos de información ecológica; 3) La cuenca contienen un río vivo de suma importancia para la ciudad de México y proveen de muchos servicios ecosistémicos y 4) la cuenca presenta varias presiones antropogénicas como la incidencia de fuego, agricultura, pastoreo y turismo ocasionados por estar inmersos en la Ciudad de México (Ávila-Akerberg et al. 2008). Sin embargo, existe una falta de información en relación al estado de conservación de la comunidad vegetal.

El objetivo de este artículo propone un planteamiento metodológico aplicado a un estudio de caso en cuatro etapas: (1) delimitación y caracterización de las unidades biofísicas basadas en condiciones ambientales, (2) cuantificación de la estructura y la composición de la vegetación, (3) clasificación de las especies de acuerdo a sus respuestas funcionales y (4) evaluación del estado de conservación basado en la estructura y la composición. Determinando una caracterización de los bosques templados heterogéneos (topográficamente) en una escala espacial adecuada y con bases ecológicas a diferencia de los métodos usados comúnmente en los inventarios nacionales forestales. Además enfatiza no sólo la importancia de la composición arbórea sino también del sotobosque caracterizando la contribución relativa de especies nativas, y especies introducidas como indicadoras del estado de deterioro.

La hipótesis principal es que las especies de malezas e introducidas se encuentran asociadas a características ambientales particulares y se distribuyen en sitios con mayor grado de disturbio, por lo tanto, son indicadoras del estado de conservación de las unidades ambientales.

Este enfoque puede proveer de herramientas para el diagnóstico de la condición de la vegetación y puede servir como base para generar estrategias de manejo y programas de restauración.

\section{MÉTODO}

Área de estudio. La cuenca el río Magdalena (19 $13^{\prime}$

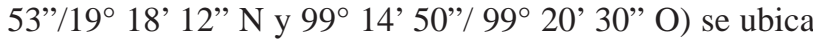
en la Sierra de las Cruces, dentro de la faja volcánica trans- 
mexicana en un intervalo altitudinal de 2.570 y $3.870 \mathrm{~m}$. La cuenca del río Magdalena se extiende dentro de la zona de conservación ecológica al sureste del Distrito Federal y cuya extensión es de 3.100 ha (figura 1). El río Magdalena es uno de los últimos ríos vivos en la ciudad de México. El río tiene una extensión de 21,6 km de longitud. Este río corre dentro del área natural $(13 \mathrm{~km})$ y luego fluye a través del área urbana. Los bosques que prevalecen en la zona son el bosque de Abies religiosa (Kunth) Schltdl. et Cham) (46\%), dominando la comunidad, seguido del bosque de Pinus hartwegii (Lindl.) (29\%), bosque de Quercus rugosa Neess - Quercus laurina Humb. et Bonpl. (8,3\%), pastizal (7,2\%) y bosques mixtos (1,3\%) (ÁvilaAkerberg et al. 2008).

Enfoque metodológico. Fue desarrollado un enfoque sistemático para determinar el estado de conservación de los bosques de la cuenca del río Magdalena (figura 2).

Delimitación y caracterización de las unidades biofísicas con base en las condiciones ambientales. Se consideraron dos componentes para delimitar las entidades ecológicas en la descripción de la cuenca (referidas a la escala de cuenca): 1) ambiente físico (geología, suelo y clima) y
2) ambiente biológico (vegetación). Se digitalizaron seis mapas de la cuenca (escala 1:25 000) del INEGI (1993). Los modelos de elevación digitales elaborados fueron utilizados para la clasificación. Las unidades biofísicas fueron delimitadas a través de fotos aéreas e imágenes de satélite. Estas unidades fueron definidas como áreas con las mismas características en términos de fisionomía, tipo de vegetación, topografía y orientación de ladera. Se realizó la fotointerpretación a 1:25 000 a través de la técnica de estereoscopia. Se describieron los atributos de las unidades biofísicas como: pendiente, orientación, tipo de bosque, uso de suelo, apertura de dosel, densidad del bosque y algunos efectos antropogénicos (presencia de pastizal o campos de cultivo en este caso). Esta información fue digitalizada, georreferenciada y orto-corregida con el método de GCPs en las imágenes de satélite en Arc View GIS 3.2 (ESRI 1999) e ILWIS 3.2 (ITC 2002) y posteriormente integrada con la información digital de topografía, geología, suelo, clima y vegetación de la cuenca.

Cuantificación de la estructura y composición de la vegetación. Se realizó un muestreo aleatorio estratificado donde se establecieron 78 parcelas de $25 \mathrm{x} 25 \mathrm{~m}$ en las 21 unidades ambientales definidas para la cuenca.

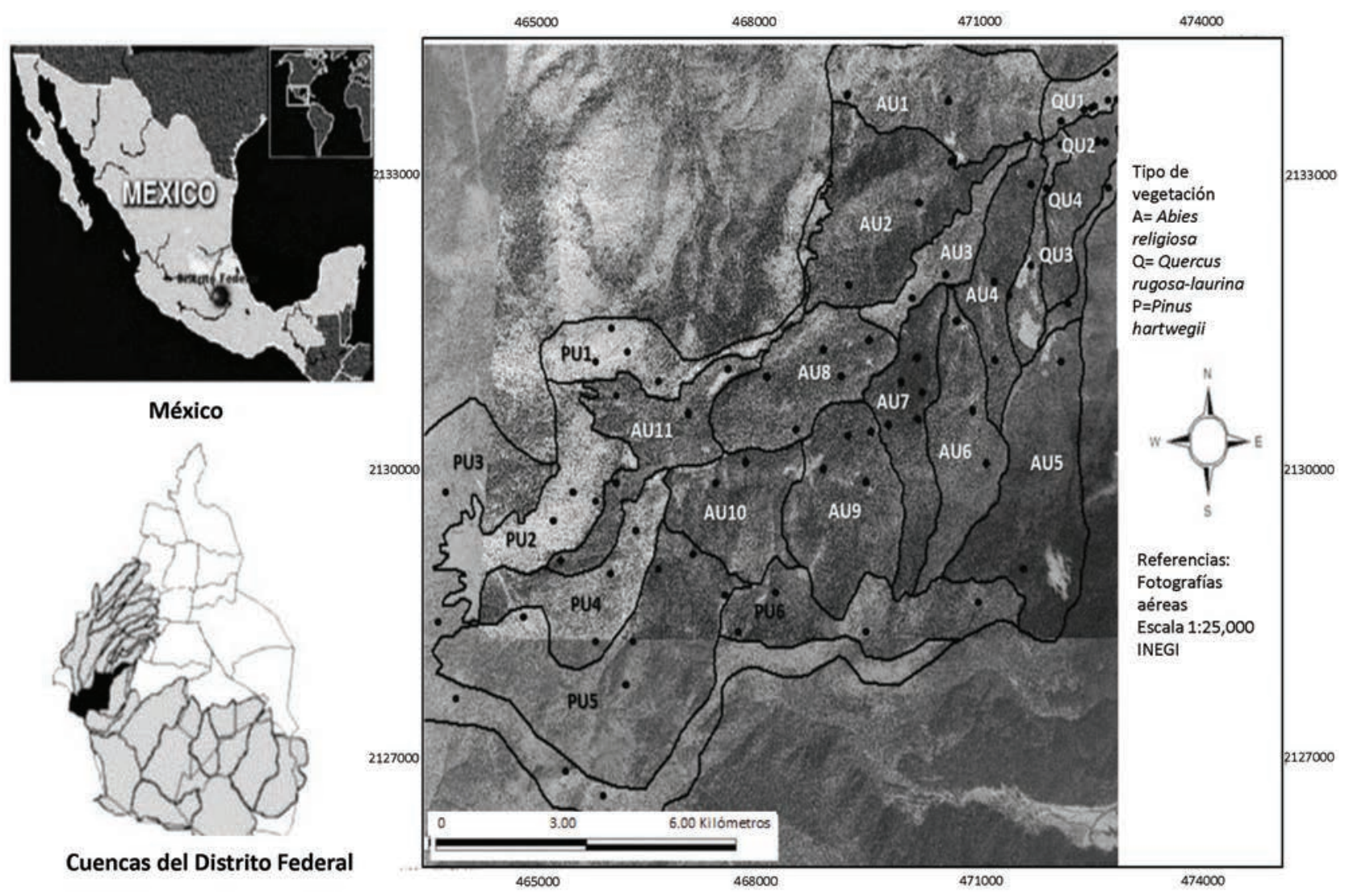

Figura 1. Ubicación de la cuenca del río Magdalena, México D. F. y sus unidades biofísicas.

Location biophysical units of Magdalena river watershed, Mexico, F.D. 


\begin{tabular}{|c|c|}
\hline $\begin{array}{l}\text { 1) Delimitación y caracterización de } \\
\text { unidades biofísicas } \\
\text {-Geología } \\
\text {-Suelo } \\
\text {-Clima } \\
\text {-Vegetación }\end{array}$ & $\begin{array}{l}\text { 2) Cuantificación de la estructura y } \\
\text { composición de la vegetación }\end{array}$ \\
\hline & $\downarrow$ \\
\hline $\begin{array}{l}\text { 4) Evaluación del estado de conservación } \\
\text {-Determinación del estado de conservación de } \\
\text { las unidades biofísicas en relación a su } \\
\text { estructura y composición (con ayuda del Índice } \\
\text { de Stenhouse). }\end{array}$ & $\begin{array}{l}\text { 3) Clasificación de la especies de acuerdo a } \\
\quad \text { sus respuestas funcionales } \\
\text {-Grupos de especies y unidades (TWINSPAN) } \\
\text {-Exploración de la presencia de gradientes } \\
\text { ambientales (DCA) } \\
\text {-Determinación de grupos funcionales y su } \\
\text { relación con el ambiente (CCA) }\end{array}$ \\
\hline
\end{tabular}

Figura 2. Marco de análisis utilizado para clasificar y caracterizar el estado de conservación de las unidades biofísicas de la cuenca del río Magdalena.

Framework used to classify and characterize the condition of biophysical units of Magdalena River, F.D.

Se calculó la riqueza y la diversidad con el índice de Shannon-Wiener a través del programa EstimateS ver-8 (Colwell 2006) para estimar la diversidad en cada unidad biofísica.

Se registraron las siguientes variables biológicas: densidad, cobertura y área basal de cada una de las especies. Se calculó el índice de valor de importancia $\left(\mathrm{IV}_{\mathrm{i}}\right)$ con la siguiente fórmula:

$$
\mathrm{IV}_{i}=\mathrm{DR}_{i}+\mathrm{Fr}_{i}+\mathrm{CR}_{i}
$$

Donde $\mathrm{DR}_{i}, \mathrm{FR}_{i}$ y $\mathrm{CR}_{i}$ representan la densidad relativa, frecuencia relativa y la cobertura relativa de las especies arbóreas y del sotobosque $i$, respectivamente, para entender el papel de las especies características, malezas y especies introducidas en cada una de las unidades biofísicas. Estas especies fueron determinadas a partir de las listas florísticas de la base de datos de CONABIO (2012).

Clasificación de las especies de acuerdo a sus respuestas funcionales. Se examinó la presencia de grupos de especies que reflejaran una respuesta similar a condiciones ambientales realizando un análisis de especies indicadoras de dos vías (TWINSPAN por sus siglas en inglés) (Hill 1994) utilizando el valor de importancia (Godefroid y Koedam 2003, LaPaix y Freedman 2010).
Se realizó un análisis de correspondencia corregido (DCA por sus siglas en inglés) (Hill 1994) para explorar si la composición de especies responde a la presencia de gradientes ambientales, realizar una interpretación visual de la agrupación de especies-sitios (unidades biofísicas) y analizar si se mantiene similar a los grupos obtenidos en el TWINSPAN (McCune y Mefford 2006).

Finalmente para determinar los grupos funcionales de respuesta, se realizó una ordenación de correspondencia canónica con el programa PC-ORD v 5.10, método de Hill's (McCune y Mefford 2006). Se utilizaron las permutaciones de Monte Carlo para determinar si los eigenvalores obtenidos con el análisis canónico fueron estadísticamente significativos $(P<0,05)$. Los coeficientes canónicos estandarizados y las correlaciones entre las variables fueron calculados entre las variables ambientales y los ejes del análisis canónico.

Evaluación del estado de conservación basado en la estructura y composición. La abundancia de malezas nativas y especies introducidas indica la modificación del hábitat y pérdida de la biodiversidad nativa (Godefroid y Koedam 2003, Stenhouse 2004). Se calculó un índice de conservación para cada unidad biofísica que consideró los valores de especies características restando el valor del índice de Stenhouse (2004) (suma de especies malezas e introducidas) con las siguientes fórmulas: 


$$
\text { Ind-S }_{i}=\mathrm{SR} / \mathrm{Nat}_{i}-\left(\mathrm{SR} / \mathrm{Mal}_{i}+\mathrm{SR} / \mathrm{Int}_{i}\right)
$$

Donde SRNat, SRMal, ${ }_{i}$, SR-Int, representan la riqueza relativa de especies características, malezas e introducidas de las unidades biofísicas $i$, respectivamente, para calcular Ind-S ${ }_{i}$ que representa el valor indicador de riqueza.

$$
\text { Ind- }-\mathrm{VI}_{i}=\mathrm{VI} / \mathrm{Nat}_{i}-\left(\mathrm{VI} / \mathrm{Mal}_{i}+\mathrm{VI} / \mathrm{Int}_{i}\right)
$$

Donde VI/Nat, VI/Mal, VI/Int ${ }_{i}$ representan el valor de importancia de especies características, malezas e introducidas de las unidades biofísicas $i$, respectivamente, para calcular Ind-VI que representa el indicador de valor de importancia.

Posteriormente, se sumaron los dos índices (UrbinaCardona y Flores-Villela 2010) y se relativizaron respecto al valor máximo obtenido para calcular un índice de conservación por unidad biofísica (Ind-Con). Finalmente, con el índice de conservación se realizó una prueba de $X^{2}$ con una prueba de bondad de ajuste para conocer si existen diferencias significativas entre las frecuencias observadas a un conjunto esperado de estas.

\section{RESULTADOS}

Delimitación y caracterización de las unidades biofísicas con base en las condiciones ambientales. Se identificaron 21 unidades ambientales en la cuenca (cuadro 1), donde cuatro corresponden al bosque de Quercus rugosa-Q. laurina, 11 al bosque de $A$. religiosa y seis al bosque de $P$. hartwegii (figura 1).

En general las unidades biofísicas del bosque de $Q$, rugosa-laurina están dominadas por especies características como Q. rugosa, Q. laurina, y Garrya laurifolia (Hartw. ex Benth.). Basadas en los atributos de la fisionomía sólo QU2 y QU4 muestran un dosel abierto y ralo con perturbaciones antropogénicas principalmente por cultivo y pastoreo.

Frecuentemente, A. religiosa fue la especie dominante en todas sus unidades aunque en algunas ocasiones asociadas a Ceanothus coeruleus (Lag.), Pinus ayacahuite (C.Ehrenb. ex Schltdl. var. (Roezl) Shaw.), G. laurifolia, Pinus montezumae (Lamb.) y P. hartwegii. También se encuentra Sambucus nigra ((L.) B.L.Turner) una maleza arbórea (Bonilla-Valencia 2014) además de que están ya establecidas algunas especies como resultado de progra-

Cuadro 1. Características principales de las unidades biofísicas de la cuenca del río Magdalena, México.

\begin{tabular}{|c|c|c|c|c|c|c|}
\hline Unidades & $\begin{array}{l}\text { Tipo de } \\
\text { suelo }\end{array}$ & $\begin{array}{l}\text { Intervalo de } \\
\text { altitud (m s.n.l.) }\end{array}$ & $\begin{array}{l}\text { Extensión } \\
\text { (ha) }\end{array}$ & $\begin{array}{l}\text { Orientación y nivel } \\
\text { de la pendiente }\end{array}$ & $\begin{array}{l}\text { Vegetación: especies } \\
\text { arbóreas dominantes }\end{array}$ & $\begin{array}{l}\text { Fisionomía y nivel de } \\
\text { conservación del bosque }\end{array}$ \\
\hline QU1 & $\begin{array}{c}\text { Ah } \\
\text { Lit/Ah }\end{array}$ & $2.700-3.000$ & 76,21 & SE/Ligera & $\begin{array}{l}\text { Quercus rugosa, (Neess), } \\
\text { Quercus laurina (Humb. et } \\
\text { Bonpl.) y Quercus crassipes } \\
\text { (Humb. et Bonpl.) }\end{array}$ & $\begin{array}{l}\text { Dosel cerrado, muy } \\
\text { denso y conservado }\end{array}$ \\
\hline QU2 & Ah/Lit & $2.700-3.100$ & 102,51 & NO/Alta & $\begin{array}{l}\text { Quercus laurina (Humb. et } \\
\text { Bonpl.) Garrya laurifolia } \\
\text { (Hartw. ex Benth.) y } \\
\text { pastizal }\end{array}$ & $\begin{array}{l}\text { Dosel abierto, poco } \\
\text { denso con signos de } \\
\text { disturbio }\end{array}$ \\
\hline QU3 & $\begin{array}{c}\text { Ah/Lit } \\
\text { Ah }\end{array}$ & $2700-3100$ & 39,43 & NO y NE/Alta & $\begin{array}{l}\text { Quercus rugosa (Neess), } \\
\text { Quercus laurina (Humb. et } \\
\text { Bonpl.) y Garrya laurifolia } \\
\text { (Hartw. ex Benth.) }\end{array}$ & $\begin{array}{l}\text { Dosel abierto, poco } \\
\text { denso semiconservado }\end{array}$ \\
\hline QU4 & $\begin{array}{c}\text { Ah/Lit } \\
\text { Ah }\end{array}$ & $2800-3200$ & 44,38 & NE y NO/Alta & $\begin{array}{l}\text { Quercus rugosa (Neess), } \\
\text { Garrya laurifolia (Hartw. } \\
\text { ex Benth.) y pastizal }\end{array}$ & $\begin{array}{l}\text { Dosel abierto, poco } \\
\text { denso con signos de } \\
\text { disturbio }\end{array}$ \\
\hline AU1 & $\mathrm{Ah} / \mathrm{Lit}$ & $2900-3400$ & 187,54 & S/Alta & $\begin{array}{l}\text { Abies religiosa (Kunth) } \\
\text { Schltdl. et Cham) y pastizal }\end{array}$ & $\begin{array}{l}\text { Dosel abierto, poco } \\
\text { denso con signos de } \\
\text { disturbio }\end{array}$ \\
\hline AU2 & $\mathrm{Ah} / \mathrm{Lit}$ & $2900-3400$ & 214,95 & S/Moderada & $\begin{array}{l}\text { Abies religiosa (Kunth) } \\
\text { Schltdl. et Cham) }\end{array}$ & $\begin{array}{l}\text { Dosel cerrado, muy } \\
\text { denso y conservado }\end{array}$ \\
\hline AU3 & Ah & $2800-3100$ & 60,19 & SE/Ligera & $\begin{array}{l}\text { Abies religiosa (Kunth) } \\
\text { Schltdl. et Cham) y } \\
\text { Ceanothus coeruleus (Lag.) }\end{array}$ & $\begin{array}{l}\text { Dosel abierto, poco } \\
\text { denso y con signos de } \\
\text { disturbio }\end{array}$ \\
\hline
\end{tabular}

Main features of biophysical units of Magdalena river basin. 


\begin{tabular}{|c|c|c|c|c|c|c|}
\hline AU4 & Ah & $3000-3100$ & 101,30 & O/Alta & $\begin{array}{l}\text { Abies religiosa (Kunth) } \\
\text { Schltdl. et Cham), Pinus } \\
\text { ayacahuite (C.Ehrenb. ex } \\
\text { Schltdl. var. (Roezl) Shaw.) } \\
\text { y Prunus serotina (Cav.) } \\
\text { McVaugh.) }\end{array}$ & $\begin{array}{l}\text { Dosel abierto, poco } \\
\text { denso y con signos de } \\
\text { disturbio }\end{array}$ \\
\hline AU5 & Ah & $3100-3400$ & 202,60 & O/Alta & $\begin{array}{l}\text { Abies religiosa (Kunth) } \\
\text { Schltdl. et Cham) }\end{array}$ & $\begin{array}{l}\text { Dosel cerrado, muy } \\
\text { denso y conservado }\end{array}$ \\
\hline AU6 & Ah & $3100-3500$ & 118,74 & E/Ligera & $\begin{array}{l}\text { Abies religiosa (Kunth) } \\
\text { Schltdl. et Cham) y Pinus } \\
\text { ayacahuite (C.Ehrenb. ex } \\
\text { Schltdl. var. (Roezl) Shaw.) }\end{array}$ & $\begin{array}{l}\text { Dosel cerrado, muy } \\
\text { denso y conservado }\end{array}$ \\
\hline AU7 & Ah & $3200-3600$ & 104,77 & O/Ligera & $\begin{array}{l}\text { Abies religiosa (Kunth) } \\
\text { Schltdl. et Cham), Garrya } \\
\text { laurifolia (Hartw. ex } \\
\text { Benth.) y Sambucus nigra } \\
\text { (L.) B.L.Turner) }\end{array}$ & $\begin{array}{l}\text { Dosel cerrado, muy } \\
\text { denso y conservado }\end{array}$ \\
\hline AU8 & Ah & $3200-3500$ & 127,55 & SE/Alta & $\begin{array}{l}\text { Abies religiosa (Kunth) } \\
\text { Schltdl. et Cham) y Pinus } \\
\text { montezumae (Lamb.) }\end{array}$ & $\begin{array}{l}\text { Dosel abierto, poco } \\
\text { denso y con signos de } \\
\text { disturbio }\end{array}$ \\
\hline AU9 & Ah & $3200-3600$ & 143,25 & N/Moderada & $\begin{array}{l}\text { Abies religiosa (Kunth) } \\
\text { Schltdl. et Cham) y pastizal }\end{array}$ & $\begin{array}{l}\text { Dosel cerrado, muy } \\
\text { denso y conservado }\end{array}$ \\
\hline AU10 & Lit/Ah & $3200-3600$ & 92,56 & E y O/Alta & $\begin{array}{l}\text { Abies religiosa (Kunth) } \\
\text { Schltdl. et Cham) y pastizal }\end{array}$ & $\begin{array}{l}\text { Dosel abierto, muy } \\
\text { denso y conservado }\end{array}$ \\
\hline AU11 & Lit/Ah & $3200-3600$ & 105,80 & $\mathrm{O}$ and E/Alta & $\begin{array}{l}\text { Abies religiosa (Kunth) } \\
\text { Schltdl. et Cham) y Pinus } \\
\text { hartwegii (Lindl.) }\end{array}$ & $\begin{array}{l}\text { Dosel abierto, denso y } \\
\text { conservado }\end{array}$ \\
\hline PU1 & $\begin{array}{c}\mathrm{Ah} / \mathrm{Ao} / \\
\mathrm{Fk}\end{array}$ & $3500-3700$ & 93,12 & SO/Alta & $\begin{array}{l}\text { Pinus hartwegii, (Lindl.) } \\
\text { y Abies religiosa (Kunth) } \\
\text { Schltdl. et Cham) }\end{array}$ & $\begin{array}{l}\text { Dosel cerrado, denso y } \\
\text { conservado }\end{array}$ \\
\hline PU2 & $\begin{array}{c}\mathrm{Ao} / \mathrm{Fh} / \\
\text { Lit }\end{array}$ & $3500-3800$ & 113,56 & E/Moderada & $\begin{array}{l}\text { Pinus hartwegii (Lindl.) y } \\
\text { pastizal }\end{array}$ & $\begin{array}{l}\text { Dosel abierto, poco } \\
\text { denso y conservado }\end{array}$ \\
\hline PU3 & $\begin{array}{l}\mathrm{Ao} / \mathrm{Fh} / \\
\text { Lit } \\
\text { Ah }\end{array}$ & $3500-3800$ & 314,53 & NE y NO/Ligera & $\begin{array}{l}\text { Pinus hartwegii (Lindl.) y } \\
\text { pastizal }\end{array}$ & $\begin{array}{l}\text { Dosel abierto, poco } \\
\text { denso y conservado }\end{array}$ \\
\hline PU4 & $\begin{array}{l}\mathrm{Ao} / \mathrm{Fh} / \\
\text { Lit } \\
\text { Lit/Ah } \\
\text { Ah }\end{array}$ & $3400-3600$ & 98,70 & NE/Moderada & $\begin{array}{l}\text { Pinus hartwegii (Lindl.) y } \\
\text { pastizal }\end{array}$ & $\begin{array}{l}\text { Dosel abierto, poco } \\
\text { denso y conservado }\end{array}$ \\
\hline PU5 & $\begin{array}{l}\text { Ao/Fh/ } \\
\text { Lit } \\
\text { Lit/Ah } \\
\text { Ah }\end{array}$ & $3400-3700$ & 247,61 & $\begin{array}{c}\mathrm{NO} / \\
\text { Moderada }\end{array}$ & $\begin{array}{l}\text { Pinus hartwegii (Lindl.) y } \\
\text { pastizal }\end{array}$ & $\begin{array}{l}\text { Dosel abierto, poco } \\
\text { denso y conservado }\end{array}$ \\
\hline PU6 & Ah & $3400-3600$ & 129,32 & NO /Ligera & $\begin{array}{l}\text { Pinus hartwegii (Lindl.) y } \\
\text { pastizal }\end{array}$ & $\begin{array}{l}\text { Dosel abierto, poco } \\
\text { denso y conservado }\end{array}$ \\
\hline
\end{tabular}


mas de reforestación (P. ayacahuite y Cuppressus lusitanica (Mill.)) y pastizales.

Las unidades del bosque de Pinus spp. siempre estuvieron dominadas por $P$. hartwegii en asociación con especies de pastizal. Estos bosques son ralos y con un dosel abierto.

Cuantificación de la estructura y composición de la vegetación. Se encontraron 192 especies en total para los tres tipos de vegetación. Este dato representa 28 \% de las 543 especies vegetales que se han reportado para la cuenca (Ávila-Akerberg et al. 2008, Santibañez-Andrade 2009). El bosque de $Q$. rugosa-laurina registró la riqueza más alta (123 especies), con 17 especies en el estrato arbóreo y 106 especies arbustivas y herbáceas. Mientras tanto el bosque de $A$. religiosa registró 98 especies con 10 en el estrato arbóreo y 88 especies arbustivas y herbáceas. En el bosque de $P$. hartwegii un total de 59 especies fueron registradas, tres de ellas fueron especies arbóreas y 56 arbustivas y herbáceas.

La riqueza osciló entre 62 y 65 especies en las unidades del bosque de Q. rugosa-laurina, QU2 mostró la ma- yor riqueza (13 árboles y 50 arbustos y hierbas; cuadro 2). El bosque de $A$. religiosa registro una riqueza de 12 a 46 especies por unidad, donde AU6 y AU7 registraron la mayor riqueza en el estrato arbóreo. Las unidades con mayor riqueza en el sotobosque fueron AU7 (41 especies), AU8 (39 especies) y AU9 (38 especies). En el bosque de P. hartwegii, la riqueza en las unidades osciló de 12 a 44 especies donde PU5 fue la unidad que registró más especies en ambos estratos (44 especies).

Las unidades del bosque de Q. rugosa-laurina tuvieron los valores más altos de diversidad, de las cuales la QU4 registró el valor más alto. Para el bosque de A. religiosa las unidades AU2, AU4, AU7 y AU8 tuvieron valores altos de diversidad en el estrato arbustivo y herbáceo pero valores bajos en el estrato arbóreo. Sólo las unidades AU6 y AU7 registraron diversidad alta en ambos estratos. Para las unidades del bosque de $P$. hartwegii la diversidad disminuyó a medida que aumenta la altitud (cuadro 2).

El número de especies de cada tipo en el bosque de Q. rugosa-laurina fue relativamente homogéneo entre las unidades. Aproximadamente 70 a $80 \%$ de toda la rique-

Cuadro 2. Riqueza (S) e índice de diversidad de Shannon-Weaner (H’) por unidad biofísica de la cuenca del río Magdalena. Ar = arbórea, $\mathrm{s}=$ sotobosque.

Richness and Shannon-Weaner diversity index per unit in Magdalena river basin. $\mathrm{Ar}=$ Tree layer, $\mathrm{s}=$ understory.

\begin{tabular}{lcccccc}
\hline Unidades & S-Ar & S-s & S-Total & H'-Ar & H'-s & H'-Total \\
\hline QU1 & 7 & 58 & 65 & 0,53 & 1,47 & 1,46 \\
QU2 & 13 & 50 & 63 & 0,78 & 1,43 & 1,52 \\
QU3 & 11 & 52 & 63 & 0,59 & 1,53 & 1,47 \\
QU4 & 9 & 53 & 62 & 0,77 & 1,60 & 1,64 \\
AU1 & 2 & 10 & 12 & 0,21 & 0,86 & 0,94 \\
AU2 & 2 & 32 & 34 & 0,14 & 1,33 & 1,35 \\
AU3 & 3 & 21 & 24 & 0,32 & 1,16 & 1,20 \\
AU4 & 4 & 37 & 41 & 0,27 & 1,32 & 1,35 \\
AU5 & 3 & 14 & 17 & 0,29 & 0,95 & 1,01 \\
AU6 & 5 & 25 & 30 & 0,60 & 1,25 & 1,33 \\
AU7 & 5 & 41 & 46 & 0,52 & 1,39 & 1,44 \\
AU8 & 3 & 43 & 0,29 & 1,31 & 1,35 \\
AU9 & 39 & 41 & 0,10 & 1,14 & 1,18 \\
AU10 & 3 & 38 & 21 & 0,18 & 1,05 & 1,10 \\
AU11 & 2 & 19 & 31 & 0,08 & 1,13 & 1,16 \\
PU1 & 3 & 29 & 0,28 & 0,97 & 1,05 \\
PU2 & 2 & 20 & 24 & 0,00 & 1,10 & 1,02 \\
PU3 & 1 & 23 & 12 & 0,00 & 1,16 & 1,12 \\
PU4 & 1 & 11 & 44 & 0,00 & 1,00 & 0,71 \\
PU5 & 1 & 41 & 24 & 0,22 & 1,25 & 1,28 \\
PU6 & 3 & & & & 1,07 & 1,09 \\
\hline
\end{tabular}


za estuvo representada por especies nativas características (40 a 46 especies), entre 10 y $20 \%$ fueron malezas nativas (12 a 15 especies por unidad) y sólo 5 a 9 \% (3 a 4 especies) fueron especies introducidas (figura 3). Las unidades QU3 y QU4 mostraron un número alto de especies nativas (45-46 especies). Las unidades QU1 y QU2 registraron más especies de malezas (15 especies), y sólo la unidad QU2 (4 especies) tuvo más especies introducidas en relación a otras unidades.

La contribución de las especies características en la comunidad a través del valor de importancia osciló entre 78,2 y 85,8 \% siendo QU3 y QU4 las unidades que mostraron los valores más altos. La unidad QU1 registró un valor de importancia alto para las malezas (16,3 \%). La unidad QU2 tuvo la mayor contribución de especies introducidas (12,6\% malezas y 10,3\% especies introducidas) (figura 3B).

Las especies con mayor valor de importancia en este bosque fueron: Solanum cervantesii (Lag.), Fuchsia mi- crophylla (Kunth.), Archibaccharis hirtella (Heering) y Smilax moranensis (M. Martens et Galeotti), especies características. Sin embargo, las unidades QU1 y QU2 presentaron a Hedera helix (L.) con valores altos y Asplenium monanthes (L.) en las cuatro unidades de este bosque con valores bajos, ambas especies introducidas. Salvia mexicana (L.) tuvo un valor alto en QU1, QU2 y QU3, Acaena elongata (L.) en QU2 y QU4, e Iresine diffusa (Humb. et Bonpl. ex Willd.) en QU1, todas malezas nativas.

La composición de las unidades del bosque de A. religiosa mostró una alta heterogeneidad. Alrededor de 63 a $88 \%$ del total fueron especies nativas características (12 a 32 especies por unidad), entre 10 y 34 \% (3 a 14 especies) fueron malezas y 2 a 8 \% (1 a 3 especies) fueron especies introducidas (figura 3A).

Las unidades AU7 y AU8 mostraron mayor número de especies características (32 y 29 especies), AU4, AU7, AU8 y AU9 mostraron mayor número de malezas (10 a 14
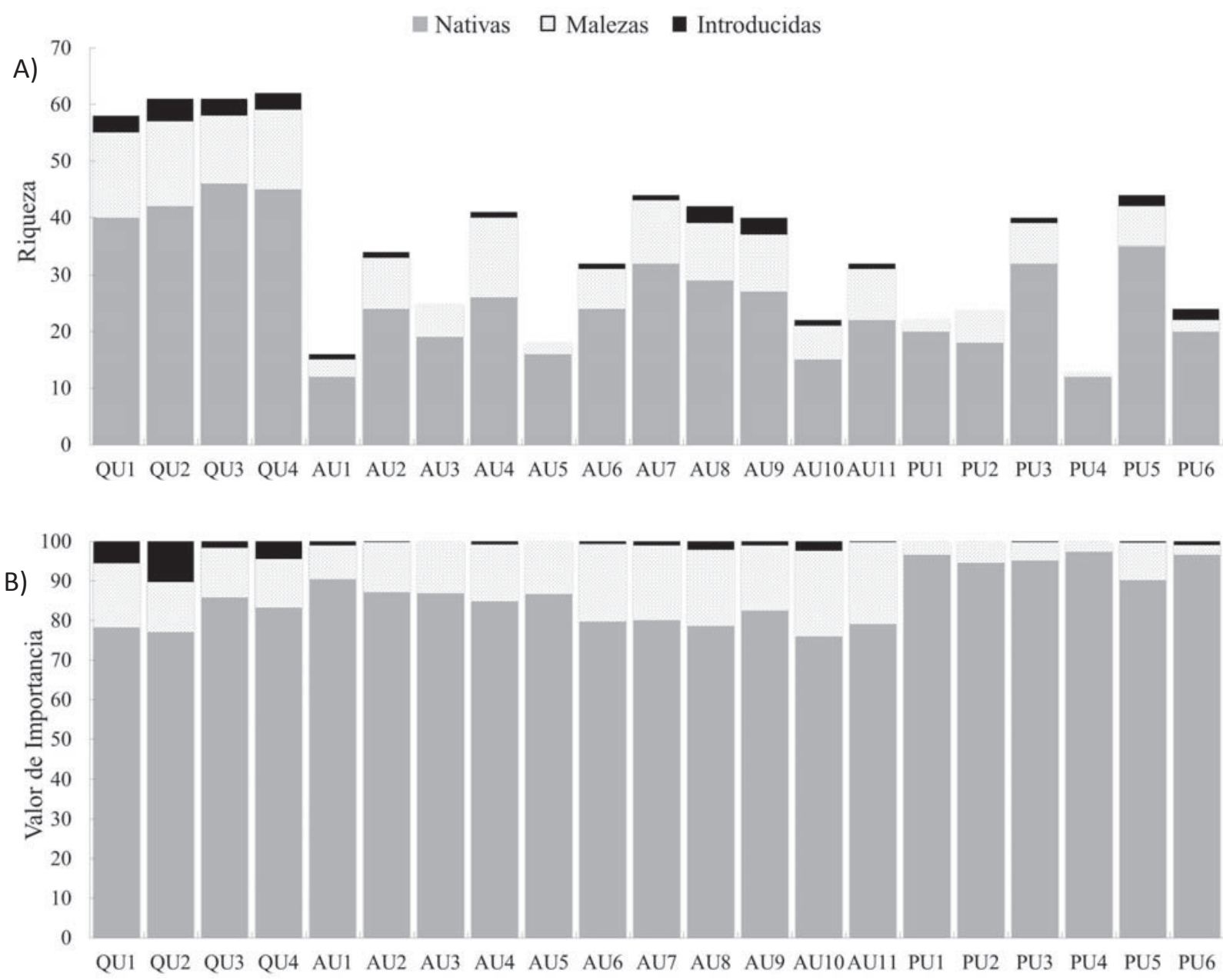

Figura 3. Riqueza (A) y valor de importancia (B) de las especies en relación a su estatus por unidad biofísica. Nativas = especies nativas características, Malezas = especies de malezas nativas, Introducidas = especies introducidas para la zona.

Richness and importance value of species regarding their origin per biophysical unit. Nativas = characteristics native species. Malezas = native weeds. Introducidas $=$ introduced species to area. 
especies) y AU8 y AU9 tuvieron mayor número de especies introducidas en relación a otras unidades (3 especies).

El valor de importancia de las especies características en esta comunidad represento alrededor de $75 \%$ a $90 \%$ siendo la unidad AU1 la de valores más altos. Las malezas en las unidades AU6, AU7, AU8, AU10 y AU11 registraron valores altos (19 a $21 \%$ ). Las unidades AU8 y AU10 tuvieron una mayor proporción de especies introducidas $(2,2$ a $2,5 \%)$ (figura 3B).

Las especies con valor más alto para todas las unidades fueron Roldana angulifolia (DC.) H.Rob. et Brettell), S. cervantesii, Roldana barba-johannis (DC.) H.Rob. et Brettell), Salvia elegans (Vahl.), y Symphoricarpos microphyllus (Kunth.) las cuales son características de este bosque. En este bosque A. elongata, maleza nativa, tuvo valores altos en la mayoría de las unidades, sobre todo en AU5, AU9, AU10 y AU11. La especie S. nigra una maleza arbórea registró valores de importancia altos en la unidad AU7. Además otras malezas están presentes en este bosque, como el caso de Alchemilla procumbens (Rose.) en AU6 y AU9, Oenothera rosea (Aiton.) en AU10, y Stellaria cuspidata (Willd. ex Schlecht.) que fue menos abundante en AU5 y AU6. La especie introducida A. monanthes se registró en la mayoría de las unidades, excepto en AU10 y AU11.

En el bosque de $P$. hartwegii alrededor de 75 a $92 \%$ del total de la composición fueron especies características (12 a 35 especies), 7 a $25 \%$ pertenecían a malezas (1 a 7 especies) y 2 a $8 \%$ fueron especies introducidas (1 a 2 especies) (figura 3A). Las unidades PU3 y PU5 registraron un mayor número de especies características (32 a 35 especies). Las unidades PU2, PU3 y PU5 registraron mayor número de malezas (6 a 7 especies). Las unidades PU5 y PU6 tuvieron un mayor número de especies introducidas (1 a 2 especies) respecto al resto de las unidades.

Las especies nativas características presentaron alrededor de 75 a $97 \%$ del valor de importancia donde PU4, PU1 y PU6 tuvieron los valores más altos. Respecto a las malezas, la unidad PU5 registró un valor alto para estas especies (9,5\%). Sólo la unidad PU6 mostró una mayor proporción de especies introducidas $(0,9 \%)$ (figura $3 \mathrm{~B})$.

En el estrato herbáceo y arbustivo las especies dominantes fueron Calamagrostis tolucensis (Kunth) Trin. ex Steud.), Alchemilla vulcanica (Cham. et Schltdl.), Muhlenbergia quadridentata (Kunth) Trin.) y Festuca tolucensis (Kunth.). En este tipo de vegetación la maleza A. elongata estuvo presente en PU5 con un valor de importancia alto y en menor proporción en PU1 y PU6. Eryngium carlinae (Delar.) es otra maleza presente en PU1, PU2 y PU3. La unidad PU6 registró dos especies introducidas Plantago major (L.) y Taraxacum officinale (Weber.), con valores de importancia bajos.

Clasificación de las especies de acuerdo a sus respuestas funcionales. En el análisis de especies indicadoras de dos vías (TWINSPAN) se formaron seis grupos funcionales de plantas (GFP) para toda la cuenca, donde 44 especies fueron especies nativas características que registraron valores de importancia altos. Estos grupos fueron sobrepuestos en un análisis de correspondencia corregido con las 21 unidades biofísicas (figura 4).

El bosque de $Q$. rugosa-laurina se dividió en dos grupos. El GFP-1 estuvo compuesto por QU1 y QU3 donde $Q$. laurina y $Q$. rugosa fueron las especies dominantes del estrato arbóreo y Salvia mexicana, Agave salmiana (Otto ex Salm-Dyck.) y S. moranensiis como malezas nativas características del sotobosque (cuadro 3). El GFP-2 estuvo compuesto por QU2 y QU4 donde G. laurifolia fue la especie arbórea dominante. En este grupo se encontró a S. nigra (una maleza arbórea), $H$. helix y A. monanthes las cuales son malezas introducidas.

En el bosque de $A$. religiosa se formaron tres grupos. El GFP-3 estuvo integrado por AU1 y AU2 donde Baccharis sordescens (DC.), Ageratina glabrata (Kunth.) R.M.King et H.Rob.) y Eupatorium schaffneri (Sch.Bip. ex Robinson.) fueron las especies características. El GFP4 estuvo integrado por AU6, AU8, AU9, AU10 y AU11 donde R. barba-johannis, Roldana albonervia (Greenm.) H.Rob. et Brettell.), Senecio callosus (Sch.Bip.), Salix paradoxa (Kunth. et C.K.Schneid.) A. elongata y O. rosea que son especies nativas tuvieron un valor de importancia alto, las dos últimas con categoría de maleza. El GFP-5 fue integrado por AU3, AU4 AU5 y AU7 y caracterizado por las especies $S$. cervantesii y Prunus serotina (Cav.) McVaugh.), y las malezas I. diffusa y Stellaria cuspidata (Willd. ex Schlecht.) tuvieron valores altos en este grupo.

El bosque de Pinus spp. se conformó en un solo grupo (GFP-6) Senecio cinerarioides (Kunth), C. tolucensis y M. quadridentata especies características, donde Eryngium carlinae (Delar.) y A. vulcanica dominan como malezas.

De acuerdo con la ordenación de los sitios, especies y las variables ambientales (figura 5), los eigenvalores de los primeros tres ejes sugieren que la separación de las unidades biofísicas por tipo de bosque está asociado a gradientes ambientales (cuadro 4). Al igual que la ordenación indirecta DCA se distinguen los mismos grupos.

Las variables más importantes que tuvieron una correlación positiva con el primer eje fueron el factor de apertura de dosel, el factor global del sitio, la luz y la altitud. Las variables que tuvieron una correlación negativa con el mismo eje fueron la humedad relativa, el suelo, la temperatura del suelo, la temperatura ambiental, el $\mathrm{pH}$ del suelo y la conductividad eléctrica. Los resultados de la prueba de permutaciones de Monte Carlo indicaron que la ordenación provee una representación significativa de la distribución de las especies y las variables ambientales registradas $(P<0,02)$.

Evaluación del estado de conservación en relación a su estructura y composición. Según los valores indicadores de conservación en relación de la riqueza y la abundancia de las especies (cuadro 5) $\left(X^{2}\right.$ calculada $=74,65$ con 


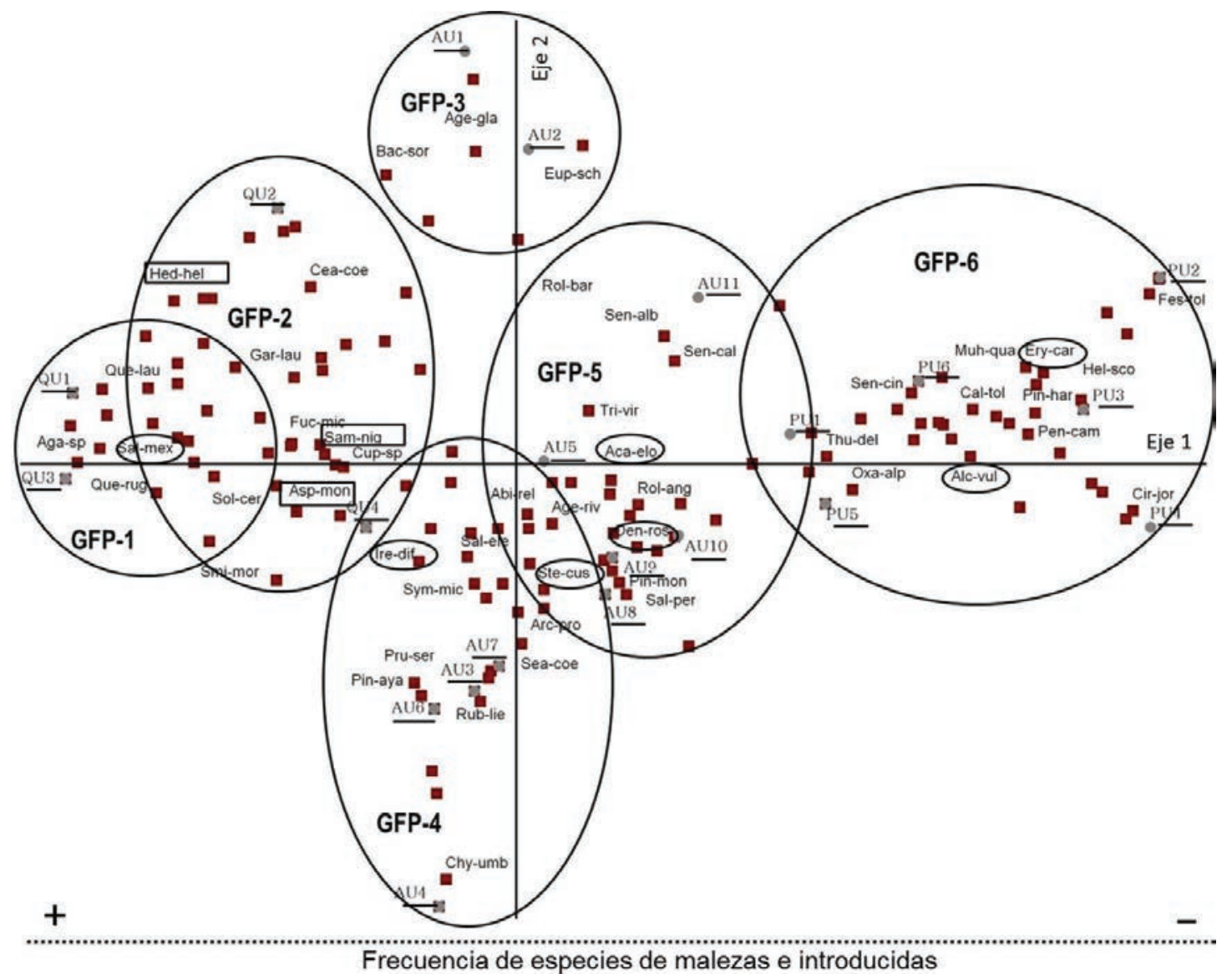

Figura 4. Resultados del análisis de correspondencia corregido de las especies y las unidades biofísicas.

Detrended correspondence analysis results for the species considered and biophysical units.

Cuadro 3. Composición de los grupos funcionales de plantas (GFP).

Composition of functional groups (GFP).

\begin{tabular}{|c|c|c|c|c|c|c|}
\hline Grupos & GFP-1 & GFP-2 & GFP-3 & GFP-4 & GFP-5 & GFP-6 \\
\hline Tipo de vegetación & $\begin{array}{l}\text { Quercus } \\
\text { rugosa-laurina }\end{array}$ & $\begin{array}{l}\text { Quercus rugosa- } \\
\text { laurina }\end{array}$ & Abies religiosa & Abies religiosa & Abies religiosa & Pinus hartwegii \\
\hline $\begin{array}{l}\text { Unidades que lo } \\
\text { componen }\end{array}$ & QU1, QU3 & QU2, QU4 & AU1, AU2 & $\begin{array}{l}\text { AU6, AU8, } \\
\text { AU9, AU10, } \\
\text { AU11 }\end{array}$ & $\begin{array}{l}\text { AU3, AU4, } \\
\text { AU5, AU7 }\end{array}$ & $\begin{array}{l}\text { PU1, PU2, } \\
\text { PU3, PU4, } \\
\text { PU5, PU6 }\end{array}$ \\
\hline Riqueza & 83 & 82 & 38 & 70 & 70 & 61 \\
\hline No. sp características & 64 & 58 & 27 & 51 & 51 & 47 \\
\hline No. sp introducidas & 3 & 5 & 1 & 1 & 4 & 2 \\
\hline No. sp malezas & 16 & 19 & 10 & 18 & 15 & 12 \\
\hline Especies dominantes & $\begin{array}{l}\text { Quercus } \\
\text { rugosa, } \\
\text { Quercus } \\
\text { laurina }\end{array}$ & $\begin{array}{c}\text { Garrya laurifolia, } \\
\text { Abies religiosa }\end{array}$ & $\begin{array}{c}\text { Abies religiosa, } \\
\text { Ageratina } \\
\text { glabrata }\end{array}$ & $\begin{array}{c}\text { Abies religiosa, } \\
\text { Rubus } \\
\text { liebmanii }\end{array}$ & $\begin{array}{c}\text { Abies religiosa, } \\
\text { Acaena } \\
\text { elongata }\end{array}$ & $\begin{array}{c}\text { Pinus } \\
\text { hartwegii, } \\
\text { Calamagrostis } \\
\text { tolucensis }\end{array}$ \\
\hline $\begin{array}{l}\text { Malezas y especies } \\
\text { introducidas }\end{array}$ & $\begin{array}{l}\text { Salvia } \\
\text { mexicana, } \\
\text { Smilax } \\
\text { moranensis }\end{array}$ & $\begin{array}{l}\text { Sambucus nigra, } \\
\text { Hedera hélix, } \\
\text { Asplenium } \\
\text { monanthes }\end{array}$ & $\begin{array}{l}\text { Salvia mexicana, } \\
\text { Asplenium } \\
\text { monanthes, } \\
\text { Iresine diffusa }\end{array}$ & $\begin{array}{c}\text { Salix paradoxa, } \\
\text { Acaena } \\
\text { elongata, } \\
\text { Oenothera } \\
\text { rosea }\end{array}$ & $\begin{array}{l}\text { Iresine diffusa, } \\
\text { Stellaria } \\
\text { cuspidata }\end{array}$ & $\begin{array}{l}\text { Eringium } \\
\text { carlinae, } \\
\text { Alchemilla } \\
\text { vulcanica }\end{array}$ \\
\hline
\end{tabular}




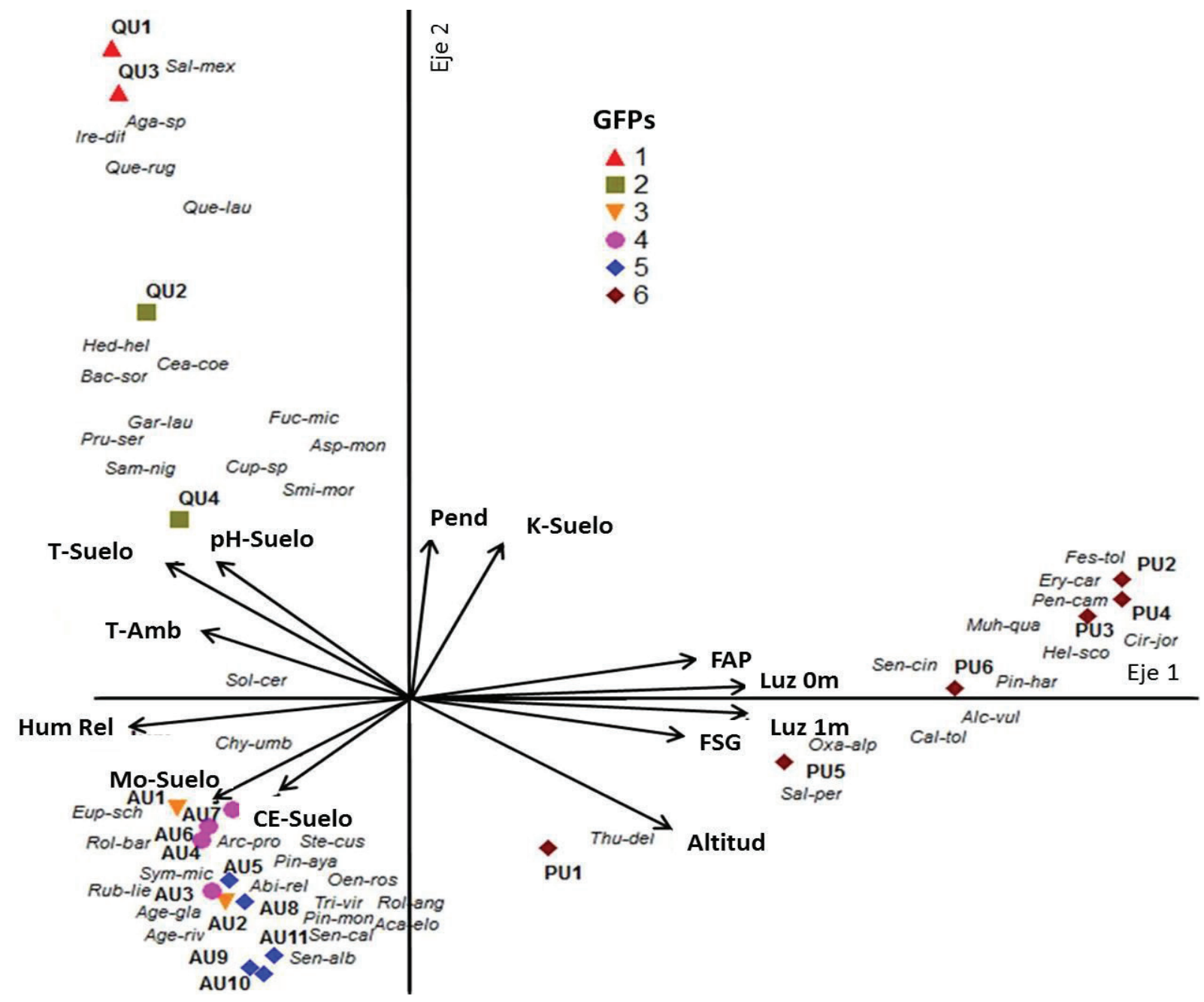

Figura 5. Análisis de correspondencia canónica de las especies y las unidades biofísicas en relación a variables ambientales de la cuenca del río Magdalena (Pend = pendiente, T-Amb = temperatura ambiental, T-Suelo = temperatura del suelo, Hum Rel = humedad relativa, Mo-Suelo = materia orgánica del suelo, $\mathrm{pH}-$ Suelo $=\mathrm{pH}$ del suelo, CE-Suelo $=$ conductividad eléctrica del suelo, K-Suelo $=$ potasio del suelo, FAP = factor de apertura de dosel, FSG = factor de sitio global, Luz 0m = luz a nivel del suelo, Luz $1 \mathrm{~m}=\mathrm{luz}$ a $1 \mathrm{~m}$ de altura.

Canonical correspondence analysis of species and environmental units in relation to environmental variables of Magdalena river basin. (Pend = slope, T-Amb = environmental temperature, T-Suelo = soil temperature, Hum Rel = relative humidity, Mo-Suelo = soil organic matter of soil, pH-Suelo = soil pH, CE-Suelo = electric conductivity of soil, K-Suelo = soil potassium, FAP = canopy openness factor, FSG = global site factor, Luz $0 \mathrm{~m}=$ light ground level, Luz $1 \mathrm{~m}=$ light level $1 \mathrm{~m}$.

Cuadro 4. Resultados del análisis canónico de correspondencia. Canonical correspondence analysis results.

\begin{tabular}{lccc}
\hline Ejes & 1 & 2 & 3 \\
\hline $\begin{array}{l}\text { Eigenvalores } \\
\text { Varianza en los datos de especies }\end{array}$ & 0,791 & 0,591 & 0,342 \\
$\quad$ \% de varianza explicada & 20,3 & 14,4 & 7,5 \\
$\quad \begin{array}{l}\text { \% de varianza explicada } \\
\quad \text { acumulada }\end{array}$ & 20,3 & 34,7 & 42,2 \\
$\begin{array}{l}\text { Correlación de Pearson } \\
\text { Especies-Ambiente }\end{array}$ & 0,997 & 0,984 & 0,993 \\
Correlación de Kendall & 0,838 & 0,848 & 0,857 \\
\hline
\end{tabular}

$\mathrm{N}=20$ y $P<0,05$ para $X^{2}$ de la tabla $=31,41$ ), se rechazó la hipótesis nula y se concluyó que hubo diferencias significativas entre las unidades en función a los valores esperados y observados.

Los resultados obtenidos a través del índice de conservación mostraron que la unidad PU4 fue la más conservada en términos de estructura y composición, seguida de la unidad PU3 del bosque de Pinus hartwegii. Para el bosque de Abies religiosa, las unidades AU1 y AU5 fueron las más conservadas; las unidades AU10, AU8 y AU4 de este tipo de bosque obtuvieron un índice de conservación muy bajo. Entre las unidades del bosque de Quercus spp., la unidad más conservada fue la QU3 y la menos conservada fue la QU2. 
Cuadro 5. Categorización de niveles de conservación de las unidades biofísicas de la cuenca del río Magdalena.

Categorization of conservation levels of biophysical units, Magdalena river basin.

\begin{tabular}{lcccccccccc}
\hline Unidades & SR/Nat & SR/Mal & SR/Int & Ind-S & VI/Nat & VI/Mal & VI/Int & Ind-VI & $\Sigma(\mathrm{S}-\mathrm{VI})$ & Ind-Con \\
\hline QU1 & 74 & 22 & 5 & 48 & 81,0 & 13,6 & 5,5 & 61,9 & 109,5 & 62 \\
QU2 & 71 & 22 & 6 & 43 & 71,9 & 19,7 & 8,3 & 43,8 & 86,6 & 49 \\
QU3 & 78 & 17 & 5 & 56 & 85,0 & 13,5 & 1,6 & 69,9 & 125,4 & 70 \\
QU4 & 74 & 21 & 5 & 48 & 79,5 & 16,3 & 4,2 & 59,0 & 107,4 & 60 \\
AU1 & 83 & 8 & 8 & 67 & 94,4 & 4,6 & 1,0 & 88,7 & 155,4 & 87 \\
AU2 & 71 & 26 & 3 & 41 & 87,2 & 12,6 & 0,3 & 74,3 & 115,4 & 65 \\
AU3 & 75 & 25 & 0 & 50 & 86,9 & 13,1 & 0,0 & 73,8 & 123,8 & 70 \\
AU4 & 63 & 34 & 2 & 27 & 84,7 & 14,4 & 0,9 & 69,4 & 96,2 & 54 \\
AU5 & 88 & 12 & 0 & 76 & 86,6 & 13,4 & 0,0 & 73,1 & 149,6 & 84 \\
AU6 & 70 & 27 & 3 & 40 & 79,5 & 19,7 & 0,7 & 59,1 & 99,0 & 56 \\
AU7 & 74 & 24 & 2 & 48 & 80,9 & 18,1 & 1,0 & 61,9 & 109,6 & 62 \\
AU8 & 67 & 26 & 7 & 35 & 78,5 & 19,3 & 2,2 & 57,0 & 91,9 & 52 \\
AU9 & 68 & 24 & 7 & 37 & 82,4 & 16,4 & 1,1 & 64,9 & 101,4 & 57 \\
AU10 & 67 & 29 & 5 & 33 & 75,9 & 21,6 & 2,5 & 51,8 & 85,1 & 48 \\
AU11 & 68 & 29 & 3 & 35 & 79,2 & 20,6 & 0,3 & 58,3 & 93,8 & 53 \\
PU1 & 86 & 14 & 0 & 73 & 96,1 & 3,9 & 0,0 & 92,2 & 164,8 & 93 \\
PU2 & 71 & 29 & 0 & 42 & 94,3 & 5,7 & 0,0 & 88,6 & 130,3 & 73 \\
PU3 & 78 & 20 & 3 & 55 & 94,7 & 5,1 & 0,2 & 89,4 & 144,4 & 81 \\
PU4 & 92 & 8 & 0 & 83 & 97,4 & 2,6 & 0,0 & 94,7 & 178,0 & 100 \\
PU5 & 75 & 20 & 5 & 50 & 89,7 & 9,9 & 0,4 & 79,4 & 129,4 & 73 \\
PU6 & 75 & 17 & 8 & 50 & 96,1 & 3,0 & 0,9 & 92,2 & 142,2 & 80 \\
\hline
\end{tabular}

$\mathrm{SR} / \mathrm{Nat}=$ Valor relativo de riqueza de especies nativas; $\mathrm{SR} / \mathrm{Ma}=$ Valor relativo de riqueza de malezas; $\mathrm{SR} / \mathrm{Int}=$ Valor relativo de riqueza de especies introducidas; Ind-S = Indicador de riqueza; VI/Nat= Valor de importancia de especies nativas; VI/Mal; Valor de importancia de malezas; VI/Int= Valor de importancia de introducidas; Ind-VI= indicador de valor de importancia; $\Sigma$ (Urbina-Cardona y Flores-Villela)= sumatoria del indicador de riqueza y el indicador de valor de importancia; Ind-Con= Índice de conservación.

\section{DISCUSIÓN}

Los resultados obtenidos a una escala general acerca del estado de conservación, utilizado en la mayoría de inventarios forestales (definido por el tipo de cobertura, la fisionomía y la densidad arbórea) arrojan resultados importantes principalmente para el estrato arbóreo (Sánchez 2004).

Por ejemplo, la presencia de especies arbóreas asociadas con las especies dominantes como en el caso de S. nigra, maleza arbórea y P. ayacahuite especie utilizada en la reforestación, así como los campos de cultivo y pastizales inducidos frecuentes en los bosques de Q. rugosa-laurina y A. religiosa, fueron signos de perturbación a esta escala.

Es útil realizar un diagnóstico a priori de la calidad de sitio a una escala general, sin embargo, al integrar atributos como composición y riqueza del sotobosque se brinda una dimensión más realista a las expectativas de conservación de los ecosistemas ya que estos pueden reflejar condi- ciones importantes dentro de la dinámica de la comunidad para el mantenimiento de la biodiversidad (Noss 1983).

En la segunda etapa de cuantificación de la estructura y composición de la vegetación se encontraron diferencias en los valores de riqueza de las unidades biofísicas (para ambos estratos), así como por tipo de vegetación (ÁvilaAkerberg et al. 2008, Santibañez-Andrade 2009). Cabe mencionar que las estimaciones de riqueza y diversidad fueron comparables por unidad ya que se contaba con la misma área muestreada por unidad, evitando así un efecto del tamaño del área. En relación a la diversidad los valores más altos del índice de Shannon-Wiener fueron para el bosque de $Q$. rugosa-laurina. Este patrón de riqueza y diversidad puede explicarse debido a que en esta área existe una mayor accesibilidad humana, por lo tanto el nivel de deterioro es alto. Lo mismo se aplica para algunas unidades del bosque de A. religiosa, cuya ubicación es accesible y tienen proximidad al río, el cual es un atractivo turístico 
en esta área. Estos picos de riqueza y diversidad en algunas unidades de la cuenca pueden ser explicadas bajo la hipótesis de disturbio intermedio (HDI) debido a que se encuentran bajo la presión de ciertos agentes de disturbio antropogénico que pueden aumentar la heterogeneidad ambiental del sistema (Shea et al. 2004). Sin embargo, es necesario un estudio que cuantifique la presencia de los factores de disturbio de manera más concisa para poder contar con mayores argumentos.

La composición es un resultado de la dinámica de la comunidad vegetal que ocurre en el sitio de estudio y que refleja su condición. La presencia de especies introducidas y malezas indican modificaciones al hábitat y pérdida de la diversidad de especies características, permitiendo así la diferenciación de sitios con diferente grado de disturbio (Stenhouse 2004). Por ejemplo, las unidades del bosque de $Q$. rugosa-laurina tuvieron un alto número de especies introducidas y malezas. Esto se debe a que este bosque está más expuesto a eventos de disturbio antropogénico, como, apertura de caminos y veredas, así como una ubicación accesible para la gente (Hobbs y Huenneke 1992). Se asumió que la presencia de estas especies puede ser explicada en base a sus rasgos de historias de vida y la tolerancia de las especies al disturbio generando patrones de distribución (Moloney y Levin 1996).

Cabe mencionar que algunas malezas presentes en este estudio ya están reportadas para otros bosques en el norte del país, como Achillea millefolium (L.) Arenaria lanuginosa (Rohrb.), Bromus carinatus (Hook. et Arn.), Geranium seemanii (Peyr.), O. rosea y Solanum nigrescens (M.Martens et Galeotti) además de algunas especies introducidas como A. monanthes y T. officinale (Encina et al. 2009).

A una escala fina, en términos de riqueza, valor de importancia de especies introducidas y malezas las unidades QU1 y QU2 tienen un bajo nivel de conservación. Es de resaltar que la unidad QU1 se consideraba conservada a una escala general pero a una escala más fina es una unidad con alto nivel de deterioro. A diferencia de las unidades QU3 y QU4 (consideradas a una escala mayor con algunos signos de deterioro) a una escala fina se encontró que son las unidades más conservadas del bosque de Q. rugosa-laurina.

En el caso del bosque de A. religiosa la unidad AU8 y AU7 tuvieron altos valores de riqueza de especies características, sin embargo, esto no es suficiente para denominarlas conservadas debido a que también presentaron una proporción alta de malezas. En el caso particular de la AU8 presentó especies introducidas. En relación a la riqueza y valores de importancia de malezas e introducidas las unidades AU6, AU7, AU8, AU9, AU10 y AU11 pueden estar siendo sometidas a un grado de disturbio alto. Esta condición puede deberse a la fácil accesibilidad y proximidad al río por lo cual existen ciertas presiones humanas. Las unidades AU1, AU3 y AU4 cuyas características a una escala general o de paisaje sugirieron signos de perturba- ción se contradice con los resultados obtenidos a una escala más fina. Por el contrario las unidades AU6 AU7, AU9 AU10 y AU11 descritas como conservadas a una escala general fueron aquellas con más presencia de malezas e introducidas denotando un nivel de disturbio alto a una escala más fina.

Sánchez-González (2004) menciona que en los bosques de A. religiosa de la faja volcánica transmexicana presentan valores similares de riqueza y composición a los reportados en este estudio. Las especies A. elongata y $A$. procumbens son malezas que se encuentran en la mayoría de los bosques de A. religiosa en México (SánchezGonzález 2004). Sin embargo A. elongata es la más abundante en todas las unidades de este bosque. Es esta especie es considerada por Rzedowski (1978) como parte de la vegetación secundaria de los bosques templados y como indicadora de perturbación según García-Romero (2002). Por lo tanto, la abundancia de esta especie puede comprometer el mantenimiento de la biodiversidad, como mecanismos de arresto de la sucesión por ser una competidora exitosa.

Es de destacar que el árbol S. nigra considerado una maleza no figura en las listas de especies características de los bosques de $A$. religiosa en la faja volcánica transmexicana. Sin embargo, en el caso de A. monanthes, una especie introducida, está bien representada en todos los bosques de la faja volcánica transmexicana (Sánchez 2004).

En el caso del bosque de $P$. hartwegii este presentó muy pocas especies introducidas y malezas. Por lo tanto las unidades se consideraron conservadas en relación a su riqueza, valor de importancia. Estas unidades se encuentran a mayor altitud lo que significa un menor impacto de las presiones antropogénicas.

En la tercera etapa acerca de la clasificación de las especies de acuerdo a sus respuestas funcionales las unidades también presentaron diferencias en su composición. Los grupos obtenidos en el análisis TWINSPAN conservaron su agregación cuando se realizó la ordenación indirecta (DCA) y en la ordenación directa (CCA), denotando que sí son grupos funcionales que están respondiendo a condiciones ambientales determinadas. Estos también indicaron la asociación de algunas unidades en relación a su composición.

Es importante reconocer que las variables ambientales más influyeron en la distribución de las especies fueron relacionadas a los recursos lumínicos. Esto debido a que en el sotobosque de los bosques templados las condiciones de luz son limitadas y el dosel del bosque se renueva a través de la dinámica de claros (Arriaga et al. 1994). Estos cambios ambientales pueden estar dados por la misma dinámica de la vegetación o puede reflejar algunos factores de perturbación (Arriaga et al. 1994).

En la cuarta etapa dedicada a estimar el estado de conservación de la vegetación en una cuenca altamente heterogénea se trató de hacer una síntesis de los resultados obtenidos para obtener indicadores de estructura y composición de la vegetación. Para ello, el nivel de infestación 
modificado de Stenhouse (2004) fue un método que nos ayudó para estimar la modificación del hábitat y pérdida de la diversidad característica. Esta disminución del valor de importancia de las especies características puede indicar cambios negativos en la diversidad local y en la calidad del hábitat (Hobbs y Huenneke 1992).

En el caso del bosque de Quercus spp donde los valores de especies de malezas e introducidas fueron altos, los valores de las especies características también fueron altos, esto minimiza su impacto en la determinación de su estado de conservación. En el caso del bosque de Abies religiosa, las unidades AU4, AU8 y AU10 tuvieron un bajo nivel de conservación, en este caso las dos primeras unidades registraron una alta riqueza de especies malezas e introducidas, sin embargo, en la unidad AU10 su bajo valor de conservación no reside en el tipo de especies que alberga sino en su valor de importancia, de aquí la importancia no sólo de estimar el número de especies sino su abundancia (LaPaix y Freedman 2010).

En la mayoría de los casos, se recomienda la planificación a escala fina sobre todo en paisajes muy heterogéneos (Von-Hase et al. 2003). Por último, la composición de la vegetación en este estudio fue un indicador importante de las condiciones ambientales del paisaje, ya que la vegetación es resultado de la interacción de los componentes ambientales y es un elemento del cual dependen directamente o indirectamente algunas funciones ecosistémicas. La importancia de este estudio radica en que la composición florística permite evaluar las prácticas de gestión posibles para fines de conservación.

\section{CONCLUSIONES}

Las 21 unidades biofísicas presentan estructura y composición diferencial que permite determinar su estado de conservación a una escala más fina. Se determinaron cinco grupos funcionales los cuales tienen una relación con la luz, temperatura, humedad y condiciones del suelo que se ordenan en un gradiente de perturbación.

La elaboración de algunos indicadores de estructura y composición basados en el nivel de infestación de Stenhouse (presencia de especies malezas e introducidas) como un estimador de la modificación del hábitat y la pérdida de la diversidad nativa dentro de las unidades biofísicas es una herramienta útil. Se puede aseverar que los bosques de la cuenca todavía mantienen estructura y composición conservada a pesar de estar inmersos en la ciudad de México, considerada una de las ciudades con mayor población en el mundo.

\section{AGRADECIMIENTOS}

Nuestro especial agradecimiento al M. en C. Oswaldo Núñez Castillo por su apoyo en campo, a Marco Antonio Romero Romero, por su apoyo en la creación de la base de datos y Ramiro Cruz Durán para la ayuda en la iden- tificación de especies de plantas. Agradecemos a la Dra. Guadalupe Barajas por su colaboración en la parte estadística de este estudio. Una parte de esta investigación fue apoyada por los proyectos PAPIIT IN202210 y PAPIME PE204209 y finalmente al Posgrado de Ciencias Biológicas de la UNAM.

\section{REFERENCIAS}

Allan JD. 2004. Landscapes and riverscapes: The Influence of Land Use on Stream Ecosystems. Annual Review of Ecology, Evolution, and Systematics 35: 257-284.

Arriaga L, S Díaz, C Mercado. 1994. Conservation of commercial management of temperature forests of Baja California Sur, Mexico. Conservation Biology 8: 1132-1140.

Ávila-Akerberg VD, B González-Hidalgo, M Nava-López, L Almeida-Leñero. 2008. Refugio de fitodiversidad en la Ciudad de México- El caso de la cuenca del río Magdalena. Journal of the Botanical Research Institute of Texas 2: 605-619.

Bonilla-Valencia L.2014. Fenología reproductive de Sambucus nigra L. en la Cuenca del río Magdalena. Tesis de Licenciatura. Universidad Nacional Autónoma de México. 76 p.

Dummel CJ, MA Pinazo. 2013. Efecto de variables de paisaje y de rodal sobre la diversidad de especies arbóreas en el sotobosque de plantaciones de Pinus taeda en la provincia de Misiones, Argentina. Bosque 34(3): 331-342.

Encina JA, A Zárate, E Estrada, J Valdés, JA Quintanilla. 2009. Composición y aspectos estructurales de los bosques de encino de la Sierra de Zapalinampe, Coahuila, México. Acta Botánica Mexicana 86: 71-108.

Eswaran H, FH Beinroth, SV Virmani. 2000. Resource management domains: a biophysical unit for assessing and monitoring land quality. Agriculture, Ecosystems and Environment 81: 155-162.

Fairbanks DHK, GA Benn. 2000. Identifying regional landscapes for conservation planning: a case study from KwaZulu-Natal, South Africa. Landscape and Urban Planning 50: 237-257.

García-Romero A. 2002. An evaluation of forest deterioration in the disturbed mountains of western Mexico City. Mountain Research and Development 22 (3): 270-277

Godefroid S, N Koedam. 2003. Distribution pattern of the flora in a peri-urban forest: an effect of the city-forest ecotone. Landscape and Urban Planning 65: 169-185.

Gomez-Mendoza L, L Galicia, R Aguilar-Santelises. 2008. Sensibilidad de grupos funcionales al cambio climático en la Sierra Norte de Oaxaca. Investigaciones Geográficas, Boletín del Instituto de Geografia, UNAM 67: 76-100.

Hill MO. 1994. DECORANA and TWINSPAN for ordination and classification of multivariate species data: a new edition, together with supporting programs, in FORTRAN 77. Huntingdon, England. Institute of Terrestrial Ecology.

Hobbs RJ, LF Huenneke. 1992. Disturbance, Diversity and Invasion: Implications for Conservation. Conservation Biology 6: 324-337.

Hooper DU, M Solan, A Symstad, S Díaz, MO Gessner. 2002. Species diversity, functional diversity, and ecosystem functioning. In Loreau M, S Naeem, P Inchausti eds. Biodiversity and ecosystem functioning - Synthesis and perspectives. New York, USA. Oxford University Press. p. 195-208. 
LaPaix R, B Freedman. 2010. Vegetation Structure and Composition within Urban Parks of Halifax Regional Municipality, Nova Scotia, Canada. Landscape and Urban Planning 98: 124-135.

Lindemayer DB, CR Margules, DB Botkin. 2000. Indicators of Biodiversity for Ecologically Sustainable Forest Management. Conservation Biology 14: 941-950.

Moloney KA, SA Levin. 1996. The effects of disturbance architecture on landscape-level population dynamics. Ecology 77: 375-394.

Mooney HA, RJ Hobbs. 2000. Invasive species in a changing world. Washington, Island Press. $457 \mathrm{p}$.

Noss RF. 1990. Indicators for Monitoring Biodiversity - A Hierarchical Approach. Conservation Biology 4: 355-364.

Parkes D, G Newell, D Cheal. 2003. Assessing the quality of native vegetation: The "habitat hectares" approach. Ecological Management \& Restoration 4: 29-38.

Pysek P, D Richardson, M Rejmánek, G Webster, M Williamson, J Kirschner. 2004. Alien plants in checklist and floras: Forwards better communications between taxonomist and ecologists. Taxon 53: 131-143.

Rzedowski J. 1978. Vegetación de México. México, D.F. México. Limusa. 432 p.

Sánchez-González A. 2004. Análisis sinecológico, florístico y biogeográfico de la vegetación del norte de la Sierra Nevada. Tesis Doctoral en Ciencias. Montecillo, México. Colegio de Postgraduados de México. $153 \mathrm{p}$

Santibañez-Andrade G. 2009. Composición y estructura de bosque de Abies religiosa en función de la heterogeneidad am- biental y determinación de su grado de conservación en la cuenca del Río Magdalena, México, D.F. Tesis de Maestría en Ciencias Biológicas. México DF, México. Universidad Nacional Autónoma de México, Facultad de Ciencias. 134 p.

Shea K, SH Roxburgh, ESJ Rauschert. 2004. Moving form pattern to process: coexistence mechanisms inder intermediate disturbance regimes. Ecological Letters 7: 491-508.

Stenhouse RN. 2004. Fragmentation and internal disturbance of native vegetation reserves in the Perth metropolitan area, Western Australia. Landscape and Urban Planning 68: 389-401.

Thackway R, A Lee, R Donohue, RJ Keenan, M Wood. 2007. Vegetation information for improved natural resource management in Australia. Landscape and Urban Planning 79: 127-136.

Urbina-Cardona JN, O Flores-Villela. 2010. Ecological-niche modeling and prioritization of conservation-area networks for Mexican herpetofauna. Conservation Biology 24: 10311041.

Westoby M, M Leishman. 1997. Categorizing plant species into functional types. In Smith TM, HH Shugart, FI Woodward eds. Plant functional types. Cambridge, UK. Cambridge University Press. p. 104-121.

Whilliams PH, CR Margules, DW Hilbert. 2002. Data requirements and data sources for biodiversity priority area selection. Journal of Biosciences (Suppl. 2) 27: 327-338.

Woodward FI. 1993. How many species are required for functional ecosystem? In Ernst-Detlef S, HA Mooney eds. Biodiversity and Ecosystem Function. Berlin, Germany. Springer-Verlang. p. 215-228. 
